\title{
Chronic Dermatomycoses of the Foot as Risk Factors for Acute Bacterial Cellulitis of the Leg: A Case-Control Study
}

\author{
Jean-Claude Roujeau ${ }^{a}$ Bardur Sigurgeirsson ${ }^{c}$ Hans-Christian Korting ${ }^{d}$ \\ Helmut Kerle Carle Paul ${ }^{b, f}$ \\ a Service de Dermatologie, Hôpital Henri-Mondor, Créteil, et bService de Dermatologie, Hôpital E.-Mueller, \\ Mulhouse, France; ' Landspitali University Hospital, Reykjavik, Iceland; dDermatological Clinic and \\ Outpatients' Clinic, University of Munich, Munich, Germany; 'Department of Dermatology and Venereology, \\ University Clinic, Graz, Austria; ${ }^{f}$ Clinical Research, Novartis Pharma AG, Basel, Switzerland
}

\section{Key Words}

Tinea pedis · Onychomycosis · Foot dermatomycosis • Bacterial cellulitis $\cdot$ Risk factors

\begin{abstract}
Objective: To assess the role of foot dermatomycosis (tinea pedis and onychomycosis) and other candidate risk factors in the development of acute bacterial cellulitis of the leg. Methods: A case-control study, including 243 patients (cases) with acute bacterial cellulitis of the leg and 467 controls, 2 per case, individually matched for gender, age ( \pm 5 years), hospital and admission date $( \pm 2$ months). Results: Overall, mycology-proven foot dermatomycosis was a significant risk factor for acute bacterial cellulitis (odds ratio, OR: $2.4 ; \mathrm{p}<0.001$ ), as were tinea pedis interdigitalis (OR: $3.2 ; \mathrm{p}<0.001$ ), tinea pedis plantaris (OR: $1.7 ; p=0.005$ ) and onychomycosis (OR: $2.2 ; p<$ 0.001 ) individually. Other risk factors included: disruption of the cutaneous barrier, history of bacterial cellulitis, chronic venous insufficiency and leg oedema. Con-
\end{abstract}

Competing Interests: B.S., J.C.R., H.C.K., H.K. and C.P. have received funds for research and been reimbursed for attending conferences by Novartis Pharma AG. C.P. is an employee of Novartis Pharma AG.

\begin{tabular}{ll}
\hline KARGER & ( ) 2004 S. Karger AG, Basel \\
1018-8665/04/2094-0301\$21.00/0 \\
$\begin{array}{l}\text { Fax + 4161306 12 34 } \\
\text { www.karger.com }\end{array}$ & $\begin{array}{l}\text { Accessible online at: } \\
\text { www.karger.com/drm }\end{array}$
\end{tabular}

clusions: Tinea pedis and onychomycosis were found to be significant risk factors for acute bacterial cellulitis of the leg that are readily amenable to treatment with effective pharmacological therapy.

Copyright $\left({ }^{2} 2004\right.$ S. Karger AG, Basel

\section{Introduction}

Acute non-necrotizing bacterial cellulitis of the leg (erysipelas) is a common infection of the skin and subcutaneous tissue causing significant morbidity [1-3]. Foot dermatomycosis (tinea pedis and onychomycosis) may constitute an important, treatable risk factor for bacterial cellulitis of the leg [4]. Fungal infection of the skin creates breaches in the epidermis and is believed to act as a portal of entry for bacterial pathogens. In the absence of other predisposing factors, clinical signs suggestive of ipsilateral athlete's foot (tinea pedis interdigitalis) have been observed in up to $80 \%$ of patients with cellulitis of the leg [5]. In $23 \%$ of patients with both tinea pedis and cellulitis, the dermatophyte infection was identified as the sole predisposing factor for cellulitis [6]. A case-control study of 167 patients with non-necrotizing bacterial cellulitis of the leg identified several risk factors, including lymphoedema, disruption of the skin barrier (mainly skin ulcer 
and toe-web intertrigo), leg oedema, venous insufficiency and being overweight, which were significantly associated with bacterial cellulitis [4]. In this study, toe-web intertrigo was only diagnosed clinically, without mycological examination.

Interestingly, the population-attributable risk (PAR) of bacterial cellulitis (i.e. the percentage of cases of bacterial cellulitis that could potentially be avoided if the risk factor was removed) was highest for toe-web intertrigo; $60 \%$ of cellulitis cases were attributable to this factor. Toe-web intertrigo is a highly prevalent condition, due primarily to fungal infection of the foot (tinea pedis); it is also frequently associated with concomitant mycological infection of the nail (onychomycosis) and of the plantar region of the foot (tinea pedis plantaris) [5-7].

The objectives of the present study were to investigate the role of mycology-proven foot dermatomycosis (tinea pedis and onychomycosis) in the development of acute bacterial cellulitis of the leg, and to identify other risk factors for the disease.

\section{Methods}

A multicentre, prospective case-control study was performed in patients with bacterial cellulitis of the leg. The protocol was approved by the appropriate institutional review boards, and all subjects gave their written informed consent to participate. Recruitment was from 30 hospitals in Austria, France, Germany and Iceland, with a minimum of 10 case patients per centre; case patients were consecutive adults admitted to either in-patient or out-patient dermatology departments for treatment of bacterial cellulitis of the leg (either first episode or recurrent). Patients were enrolled as cases if they experienced an acute (within $48 \mathrm{~h}$ ) onset of signs and symptoms of bacterial cellulitis, consisting of well-demarcated plaque-like cutaneous lesions, characterized by localized erythema, increased warmth and swelling, and associated with fever $>38^{\circ} \mathrm{C}$ and/or chills. In all cases, a dermatologist clinically validated the diagnosis of acute bacterial cellulitis of the leg. Patients were not enrolled if they had bilateral cellulitis, abscesses or necrotizing fasciitis of the leg, or if they had used topical antifungal agents within 1 week or systemic antifungal agents within 4 weeks before admission.

Control subjects were patients admitted to the same hospital, with an acute condition. Two controls were enrolled per case, individually matched for age ( \pm 5 years), gender, hospital and date of admission ( \pm 2 months of the case patient). Exclusion criteria for concomitant conditions and antifungal treatments were similar to those for cases.

All data were obtained during a single visit by a dermatologist who recorded the patient's medical history and examined the feet for clinical signs of dermatomycosis. The severity of signs of tinea pedis interdigitalis (scaling, fissuring and maceration) was rated for each toe-web on both feet, and signs of tinea pedis plantaris (scaling, fissuring and blistering) were evaluated on the soles of both feet. Each disease sign was rated on a 4 -point scale $(0=$ none, $1=$ mild, $2=$ moderate, $3=$ severe). Toenails from all toes, with the exception of the fifth (smallest) toe, were assessed on both feet for the presence or absence of clinical signs suggestive of onychomycosis.

Clinically significant signs of foot dermatomycosis were determined from clinical examination of the target leg. Clinically significant suspicion of tinea pedis interdigitalis was defined as at least 1 toe-web on the target leg with a score of at least 2 for scaling and/or fissuring (maceration was not included as it is non-specific). Clinically significant suspicion of tinea pedis plantaris was defined as a plantar region on the target leg with a score of at least 2 for clinical signs, i.e. scaling, fissuring and blistering. Clinically significant suspicion of onychomycosis was defined as the presence of nail abnormalities suggestive of onychomycosis on at least one toenail on the target leg.

Mycological samples were obtained from the foot of the affected leg in cases, and the corresponding foot in controls. Two samples were taken from each of the three sites: interdigital, plantar and nail. If signs of dermatomycosis were present, samples were taken from the most severely affected site; otherwise, samples were obtained from the third and fourth interdigital spaces, 2 different locations on the plantar region and from 2 toenails (including the big toe). Samples were sent to a central laboratory and evaluated by personnel unaware of their origin. Specimens were examined using $\mathrm{KOH}$ wet mounts for the presence of hyphae and were cultured in Sabouraud dextrose agar. The inoculated plates were incubated at a temperature of $28^{\circ} \mathrm{C}$ and assessed for the first time after 2 weeks. Cultures were confirmed negative following re-examination after a further 4 weeks. Patients with a positive result were referred to their general practitioner for treatment.

\section{Characterization of Other Potential Risk Factors}

Other risk factors such as being overweight (defined as body mass index $\geq 27$ ) [8], alcohol/smoking history and previous medical conditions as reported by the patient were also recorded. Patients were examined for the presence of potential risk factors such as venous insufficiency, leg ulcer and abolition of peripheral pulse or leg oedema.

\section{Statistical Analysis}

All demographic and background characteristics were based on the 'all subjects population' defined as all subjects (cases and controls) from whom data were available and who had signed the informed consent.

The analysis population included 'set subjects' defined as all subjects (cases and controls) from whom data were available, who had signed the informed consent and who were part of a set of 1 case and at least 1 control.

The primary evaluation was performed according to a 2-step hierarchical testing procedure, firstly to demonstrate an association between dermatomycosis on the target leg and bacterial cellulitis and, secondly, between tinea pedis interdigitalis and bacterial cellulitis. The second test was only analysed if the first test showed significance [limits of $95 \%$ confidence interval (CI) of odds ratio (OR) not including 1]. The OR was estimated using standard methods of analysis of matched data [9]. All ORs were presented together with the 95\%, two-sided CIs. For this primary analysis, only the results of mycological culture were taken into account as this was considered proof of a diagnosis of foot mycosis. However, OR for positive mycology as defined by positive direct microscopy and/or positive mycological culture was also determined. 
Fig. 1. Prevalence of foot dermatomycosis, as defined by positive mycology (positive culture and/or microscopy).

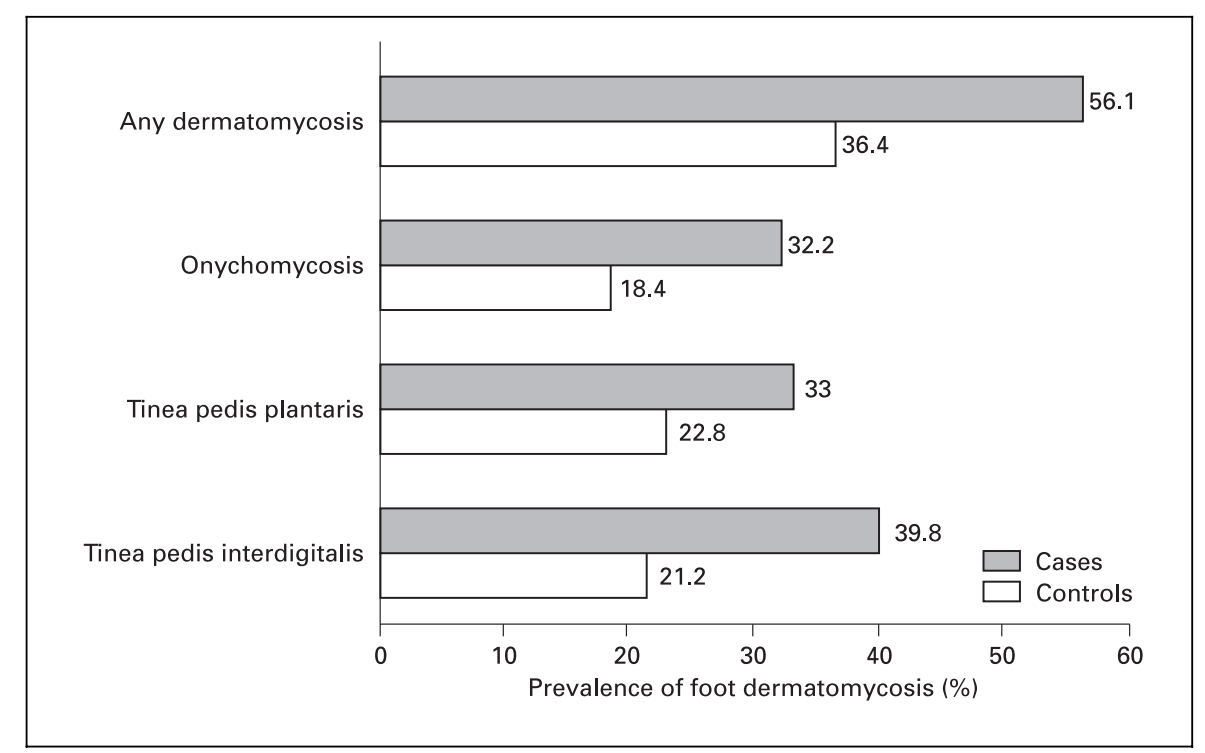

The secondary evaluation examined the association between bacterial cellulitis and subtypes of dermatomycosis using the same methodology as above. For this evaluation, the mycology results were taken into account. Additionally, a multivariate model was set up to further investigate the possible influence of other risk factors. Conditional logistic regression was applied to this analysis using a forward-step selection method. Possible covariates were chosen from previous univariate analyses with $p<0.1$. Individual score tests were used to select risk factors for the model. At each step, risk factors were added to the model in order of significance (the variable with the largest $\chi^{2}$ value was added to the model). The step was repeated, adjusting for variables already in the model, until all significant variables were included. A factor was considered significant if $\mathrm{p} \leq 0.05$ at that step.

The PAR estimates the proportion of disease cases that could be prevented if risk factors were reduced or eliminated. The PAR was calculated for dermatomycosis, tinea pedis plantaris and onychomycosis, as well as for each of the significant risk factors determined from the multivariate analysis. The control group was assumed to be representative of the general population, so the prevalence of each risk factor in the general population was derived from its prevalence in the control.

The PAR was therefore calculated as:

$$
\mathrm{PAR} \%=\frac{\mathrm{P}(\mathrm{RR}-1)}{\mathrm{P}(\mathrm{RR}-1)+1} \times 100
$$

where PAR is the population-attributable risk, $\mathrm{P}$ is the prevalence of the factor in the control group and RR is the relative risk.

The required sample size was calculated from an estimated overall prevalence of $\geq 20 \%$ for dermatomycosis and $\geq 15 \%$ for tinea pedis interdigitalis, a common OR of 2 , a correlation of exposure between a case and 1 control patient of 0.2 and a two-sided $5 \%$ significance level. The overall statistical power of the hierarchical testing procedure was at least $82 \%$. The sample size calculation was performed using PASS ${ }^{\odot} 6.0$ software (NCSS Statistical Software, Kaysville, Utah, USA; matched case-control module). No adjustments for drop-outs were required as this was a 'one-visit' study.

Tinea pedis and Onychomycosis as Risk

Factors in Acute Bacterial Cellulitis

\section{Results}

The total population (all subjects) on which demographic and background characteristics were based consisted of 243 cases and 467 controls. The set subject population (with at least 1 matched control for each case) on which all the analysis was performed included 228 cases and 439 controls.

Mean ages in cases and controls were 59 and 61 years, respectively, and the male-to-female ratio was approximately 50:50. Cases and controls were similar demographically and with regard to most other baseline factors.

\section{Mycology-Proven Foot Dermatomycosis among Cases and Controls}

The prevalence of any foot dermatomycosis, tinea pedis interdigitalis, tinea pedis plantaris or onychomycosis defined by positive mycology, i.e. positive culture or positive direct microscopic examination, was considerably higher in cases $(56.1,39.8,33.0$ and $32.2 \%$, respectively) than in controls $(36.4,21.2,22.8$ and $18.4 \%$, respectively; fig. 1). The same imbalance was apparent if the analyses were restricted to positive cultures for dermatophytes, yeasts or moulds. Culture-positive foot dermatomycosis and tinea pedis interdigitalis were found in 42.5 and $29.8 \%$ of cases compared with 24.1 and $13.3 \%$ of controls, respectively.

As expected, the majority of cultures (67\%) was positive for a dermatophyte. Yeasts were the second most

Dermatology 2004;209:301-307 


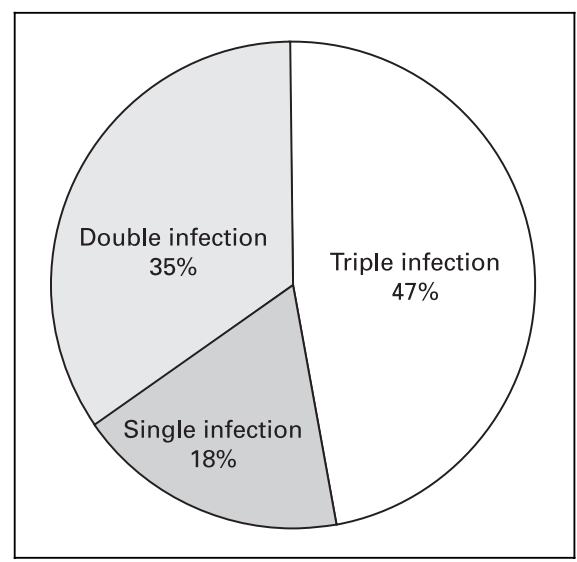

Fig. 2. Fungal infections in patients with bacterial cellulitis of the leg and foot dermatomycosis. Single infection: onychomycosis or tinea pedis plantaris or tinea pedis interdigitalis alone; double infection: onychomycosis and tinea pedis plantaris or tinea pedis interdigitalis, or tinea pedis plantaris and tinea pedis interdigitalis; triple infection: onychomycosis, tinea pedis plantaris and tinea pedis interdigitalis.

Table 1. Univariate OR of foot dermatomycosis, defined by positive mycology, in cases and controls

\begin{tabular}{|c|c|c|}
\hline & Univariate OR & $95 \% \mathrm{CI}$ \\
\hline Any dermatomycosis & $2.4^{* *}$ & $1.7-3.3$ \\
\hline Tinea pedis interdigitalis & $2.8^{* *}$ & $1.9-4.2$ \\
\hline Tinea pedis plantaris & $1.7^{*}$ & $1.2-2.4$ \\
\hline Onychomycosis & $2.2 * *$ & $1.5-3.3$ \\
\hline
\end{tabular}

$* \mathrm{p}=0.005 ; * \mathrm{p}<0.001$

prevalent organism. Amongst dermatophytes, Trichophyton dermatophytes (T. rubrum and T. mentagrophytes) were the most prevalent organisms cultured from all the sites sampled in both groups.

Culture-proven dermatomycosis was shown to be a significant risk factor for bacterial cellulitis (OR: 2.4, 95\% CI: $1.7-3.5, \mathrm{p}<0.001)$; this was also shown for tinea pedis interdigitalis (OR: 3.2, 95\% CI: 2.0-5.0, p < 0.001). The magnitude of the risk (OR) was similar when evaluated by positive mycology (defined as positive culture and/or microscopy; table 1). Mycology-proven tinea pedis plantaris and onychomycosis were also identified as significant risk factors for bacterial cellulitis (table 1).

Among patients with bacterial cellulitis and foot dermatomycosis, as determined by positive mycology, $82 \%$ had fungal infections at several sites (fig. 2). Almost half

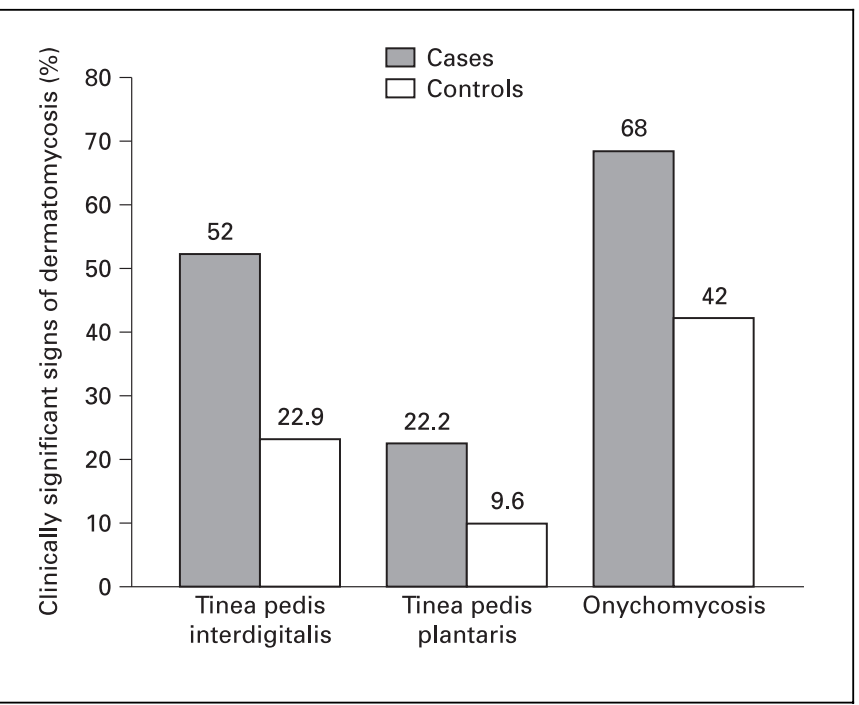

Fig. 3. Prevalence of clinically significant signs suggestive of dermatomycosis.

$(47 \%)$ of these patients had a triple infection and more than one third had a double infection, indicating that most patients had widespread foot mycosis.

\section{Severity of Signs of Dermatomycosis}

Tinea pedis interdigitalis. Patients with bacterial cellulitis had a significantly higher mean number of affected interdigital spaces than controls (2.6 and 1.4, respectively; $\mathrm{p}<0.001)$ and had significantly higher scores for scaling (cases: mean $=3.6$; controls: mean $=1.7$ ), maceration (cases: mean $=1.8$; controls: mean $=0.7$ ), fissuring (cases: mean $=1.7$; controls: mean $=0.5$ ) and total disease severity (cases: mean $=7.1$; controls: mean $=2.9$ ), compared with controls $(\mathrm{p}<0.001)$.

Tinea pedis plantaris. The signs of tinea pedis plantaris (scaling, fissuring and blistering) were severer in cases, compared with controls. The difference in terms of signs and symptoms displayed on the target leg between groups (cases: mean $=0.9$, median $=1.0$; controls: mean $=0.4$ ) was statistically significant $(\mathrm{p}<0.001)$.

Onychomycosis. In patients with onychomycosis, the mean number of toenails affected on the target leg was greater in cases (mean $=1.7$ toenails affected) compared with controls $($ mean $=0.9 ; \mathrm{p}<0.001)$.

\section{Clinically Significant Signs of Dermatomycosis}

The prevalence of clinically significant signs of tinea pedis interdigitalis, tinea pedis plantaris and onychomycosis was higher in cases than controls (fig. 3 ). 
Table 2. Non-mycological risk factors

\begin{tabular}{|c|c|c|c|c|}
\hline & \multirow[t]{2}{*}{ OR } & \multirow[t]{2}{*}{$95 \% \mathrm{CI}$} & \multicolumn{2}{|c|}{ Prevalence, $\%$} \\
\hline & & & $\begin{array}{l}\text { cases } \\
(\mathrm{n}=243)\end{array}$ & $\begin{array}{l}\text { controls } \\
(\mathrm{n}=467)\end{array}$ \\
\hline History of bacterial cellulitis & 29 & $11.7-72$ & 34.0 & 1.0 \\
\hline Disruption of cutaneous barrier & 18.2 & $9.2-36.2$ & 39.0 & 4.5 \\
\hline Ulcer on the target leg & 9 & $3.7-21.8$ & 12.0 & 1.5 \\
\hline Chronic leg oedema & 8.5 & $4.3-16.9$ & 21.0 & 4.0 \\
\hline History of deep venous thrombosis & 5.6 & $2.5-12.6$ & 10.0 & 3.0 \\
\hline Varicose eczema & 4.6 & $1.4-14.7$ & 4.5 & 1.5 \\
\hline Abolition of peripheral pulse & 2.9 & $1.3-6.1$ & 9.0 & 3.0 \\
\hline Hyperpigmentation & 2.9 & $1.8-4.5$ & 25.5 & 12.0 \\
\hline Being overweight & 2.85 & $2.0-4.0$ & 62.6 & 37.2 \\
\hline History of venous insufficiency & 2.8 & $1.9-4.1$ & 49.0 & 27.0 \\
\hline History of varicose veins & 2.2 & $1.5-3.3$ & 40.0 & 24.0 \\
\hline History of venous leg surgery & 1.8 & $1.05-3.2$ & 12.0 & 7.0 \\
\hline
\end{tabular}

Table 3. Multivariate risk factors for bacterial cellulitis of the leg

\begin{tabular}{lccc}
\hline & Adjusted OR & $\begin{array}{l}\text { Prevalence } \\
\text { in controls, } \% 1\end{array}$ & $\begin{array}{l}\text { PAR } \\
\%\end{array}$ \\
\hline Disruption of the cutaneous barrier & $22.0(9.4-51.5)$ & 4.5 & 48.5 \\
Being overweight & $2.8(1.6-5.0)$ & 37.2 & 40.7 \\
Tinea pedis interdigitalis & & 13.3 & 22.5 \\
History of bacterial cellulitis & $3.2(1.6-6.3)$ & 1.1 & 20.2 \\
Chronic leg oedema & $24.0(7.1-81.2)$ & 4.3 & 13.2 \\
\hline
\end{tabular}

Figures in parentheses indicate $95 \%$ CI.

1 An estimate of prevalence in the population.

2 Positive culture only.

\section{Additional Univariate Risk Factors}

In the univariate analysis, additional significant risk factors for bacterial cellulitis were: history of bacterial cellulitis of the target leg, disruption of the cutaneous barrier and being overweight (table 2). The most frequent causes of disruption of the cutaneous barrier other than foot dermatomycosis and venous leg ulcers were wounds and pressure ulcers. Smoking (27.1\% of controls and $22.4 \%$ of cases), alcohol consumption (5.5\% of controls and 5.7\% of cases), a history of atherosclerosis on the target leg (3.0\% of controls and $4.8 \%$ of cases) or diabetes $(12.5 \%$ of controls and $13.2 \%$ of cases) were not identified as significant risk factors.

\section{Conditional Logistic Regression Analysis}

Significant $(\mathrm{p}<0.1)$ univariate risk factors incorporated in the multivariate model were: foot dermatomyco- sis, i.e. tinea pedis interdigitalis, onychomycosis, tinea pedis plantaris (all defined by positive mycology); being overweight; history of bacterial cellulitis; history of venous insufficiency; varicose veins; deep vein thrombosis; venous leg surgery; leg ulcer; venous insufficiency; hyperpigmentation; disruption of the cutaneous barrier (wound, pressure ulcer); abolition of peripheral pulse and chronic leg oedema.

Risk factors that remained independently associated with bacterial cellulitis in the multivariate analysis are listed in table 3 . Tinea pedis interdigitalis was amongst the 5 variables that remained independently associated with bacterial cellulitis. Other factors with the most influence on risk of bacterial cellulitis were: signs and symptoms of disruption of the cutaneous barrier on the target leg; history of bacterial cellulitis on the target leg; chronic leg oedema of the target leg, and being overweight. 
The PAR was estimated for each risk factor that remained significant in the multivariate analysis (table 3). Interestingly, 2 preventable risk factors, i.e. overweight and tinea pedis interdigitalis, were associated with 40.7 and $22.5 \%$ of the cases of acute bacterial cellulitis of the leg, respectively.

\section{Discussion}

This study demonstrated a significant association between dermatomycosis of the foot and acute bacterial cellulitis of the leg. The prevalence of mycology-proven foot dermatomycosis was higher in patients with bacterial cellulitis than in controls, with the majority of patients having fungal infections at several sites. Both the extent and severity of signs of dermatomycosis were more pronounced in cases, compared with controls. The different types of dermatomycosis (i.e. tinea pedis interdigitalis, tinea pedis plantaris and onychomycosis) were each, separately, found to be significant risk factors for bacterial cellulitis. Since mycological samples were obtained during the course of acute cellulitis and since there is no published evidence that systemic antibiotics are associated with other mycoses than gastrointestinal tract candidiasis and napkin dermatitis, there is no reason to suspect that dermatomycosis was a complication of antibiotic therapy. Additional risk factors for acute bacterial cellulitis were: disruption of the cutaneous barrier, being overweight, signs of venous insufficiency and chronic leg oedema.

Similar to the results of the study of Dupuy et al. [4], this study did not identify diabetes as a risk factor, which appears to contradict current clinical experience [10]. However, it can be hypothesized from this study and the study by Dupuy et al. [4] that most cases of cellulitis in diabetic patients could not be included. Indeed, bacterial cellulitis in diabetic patients usually manifests as a subacute infection, often with abscess or drainage, and therefore did not meet the diagnostic criteria of acute bacterial cellulitis (erysipelas) without abscess required for inclusion in the present study.

The results presented here are consistent with the study by Dupuy et al. [4] but the present study has several strengths. A larger sample size was included in this study $(\mathrm{n}=710)$ compared to Dupuy et al. $(\mathrm{n}=461)$. However, the most notable difference is the absence of a laboratory diagnosis in the study of Dupuy et al. [4]. In this study, both microscopy and culture analyses were undertaken to confirm the presence of foot dermatomycosis.
Both studies identified disruption of the cutaneous skin barrier, leg oedema, venous insufficiency and being overweight as significant risk factors associated with bacterial cellulitis of the leg. The PAR for toe-web intertrigo was higher in the study by Dupuy et al. [4], compared to culture-proven tinea pedis in the present study (60 vs. $22.5 \%$, respectively). The lower sensitivity of the microbiological methods used for assessing tinea pedis interdigitalis may account at least in part for this discrepancy. It has been estimated that $30-50 \%$ of patients with dermatomycosis fail to show positive mycology $[7,11]$. If the sensitivity of mycological culture in the present study is assumed to be only $60 \%$ in both cases and controls, the true prevalence of culture-proven tinea pedis interdigitalis in controls would be $22 \%$, which would translate into a PAR of $33 \%$ for tinea pedis interdigitalis.

PAR was high for overweight patients and those with a history of bacterial cellulitis. The PAR was highest for disruption of the cutaneous barrier, thus emphasizing that the presence of a portal of entry for bacteria is a predisposing factor for bacterial cellulitis.

Amongst risk factors identified in the multivariate analysis, tinea pedis interdigitalis is the most amenable to therapeutic intervention. Although foot dermatomycosis is highly prevalent, only a minority of patients with dermatomycosis is treated $[12,13]$. The disease may be asymptomatic, and patients who do not experience complications may not seek medical advice. Furthermore, the medical complications associated with foot dermatomycosis may be underestimated. This study highlights the importance of diagnosing and treating foot dermatomycosis, since it is an easily treatable risk factor for bacterial cellulitis. Given the fact that most patients suffer from infection at multiple sites including interdigital spaces, plantar region and nail, careful sampling and specialized care are appropriate for treatment selection and followup.

\section{Conclusion}

Results from this study demonstrate that foot dermatomycoses (i.e. tinea pedis and onychomycosis) are significant risk factors for bacterial cellulitis of the leg. As these represent risk factors that are the most amenable to treatment, careful screening and treatment of foot dermatomycoses can help eliminate these specific risk factors for bacterial cellulitis of the leg, especially in patients with other risk factors, such as history of this disease, chronic venous insufficiency and being overweight. 


\section{Acknowledgements}

We thank the following contributors: Austria: Prof. Dr. P. Fritsch, Dr. C. Hoffmann, Dr. M. Kaulich, Prof. D. Kopera, Dr. N. Reider, Prof. Dr. A. Steiner; France: Dr. K. Angeli, Prof. P. Bernard, Prof. J.-M. Bonnetblanc, Dr. S. Boulinguez, Dr. C. Catala, Dr. A.-M. Causeret, Prof. A. Claudy, Dr. P. Combemale, Dr. E. Crespel, Dr. B. Cribier, Prof. B. Crickx, Dr. H. Dega, Prof. L. Dubertret, Dr. O. Estines, Prof. Dr. J.-J. Grob, Dr. N. Ismaili, Prof. P. Joly, Dr. D. Jullien, Prof. J.-P. Lacour, Dr. S. Lagrassa, Dr. E. Mahé, Dr. C. Mar- quart, Prof. J.-P. Ortonne, Dr. B. Roth, Dr. B. Sassolas, Dr. B. Schubert, Prof. L. Vaillant, Dr. P. Young; Germany: Prof. Dr. C. Bayer, Dr. C. Borelli, Prof. Dr. J. Brasch, Prof. Dr. I. Effendy, Dr. K. Friebe, Dr. E. Glorer, Prof. Dr. U.-F. Haustein, Dr. M. Hoffmann, Prof. Dr. K. Hoffmann, Dr. K. Kluge, Dr. H. Loeffler, Dr. P. Nenoff, Dr. K. Niedergethmann, Dr. F. Noack-Wiemers, Dr. A. Pfister-Wartha, Prof. Dr. E. Schoepf, Prof. Dr. R. Schopf; Iceland: Dr. J.H. Olafsson, Dr. B. Sveinsson; Novartis/Switzerland: S. Emady Azar, Dr. C. McGeown, R. Schweizer; Parexel/Germany: K. Hirsch, Y. Limbach.

\section{References}

1 Stulberg DL, Penrod MA, Blatny RA: Common bacterial skin infections. Am Fam Physician 2002; 66:119-124.

2 Ronnen M, Suster S, Schewach-Millet M, Modan M: Erysipelas: Changing faces. Int J Dermatol 1985;24:169-172.

3 Guberman D, Gilead LT, Zlotogorski A, Schamroth J: Bullous erysipelas: A retrospective study of 26 patients. J Am Acad Dermatol 1999;41:733-737.

4 Dupuy A, Benchikhi H, Roujeau JC, Bernard P, Vaillant L, Chosidow O, Sassolas B, Guillaume JC, Grob JJ, Bastuji-Garin S: Risk factors for erysipelas of the leg (cellulitis): Casecontrol study. BMJ 1999;318:1591-1594.
5 Semel JD, Goldin H: Association of athlete's foot with cellulitis of the lower extremities: Diagnostic value of bacterial cultures of ipsilateral interdigital space samples. Clin Infect Dis 1996;23:1162-1164.

6 Roldan YB, Mata-Essayag S, Hartung C: Erysipelas and tinea pedis. Mycoses 2000;43:181183.

7 Leyden JJ, Kligman AM: Interdigital athlete's foot: The interaction of dermatophytes and resident bacteria. Arch Dermatol 1978;114: 1466-1472.

8 NIH: The practical guide: Identification, evaluation and treatment of overweight and obesity in adults. www.nhlbi.nih.gov

9 Breslow NW, Day NE: Statistical Methods in Cancer Research. Lyon, International Agency for Research on Cancer Scientific Publications, 1980, vol 1: The analysis of case-control studies.
10 Suss SJ, Middleton DB: Cellulitis and related skin infections. Am Fam Physician 1987;36: 126-136.

11 Hull PR, Gupta AK, Summerbell RC: Onychomycosis: An evaluation of three sampling methods. J Am Acad Dermatol 1998;39:10151017.

12 Roberts DT: Prevalence of dermatophyte onychomycosis in the United Kingdom: Results of an omnibus survey. Br J Dermatol 1992;126: 23-27.

13 Sais G, Jucgla A, Peyri J: Prevalence of dermatophyte onychomycosis in Spain: A cross-sectional study. Br J Dermatol 1995;132:758761. 